\title{
Conceptual Scheme of Motor Asymmetry Control in Aerobic Cyclic Sports
}

\author{
Svetlana S. Khudik, Aleksandr I. Chikurov, \\ Andrey D. Burmistrov and Aleksandr L. Voinich* \\ Siberian Federal University \\ Krasnoyarsk, Russian Federation
}

Received 30.10.2020, received in revised form 24.12.2020, accepted 28.01.2021

\begin{abstract}
In this article, based on well-known literary data, the motor asymmetry of a person in the training process of aerobic cyclic sports is considered. The authors have analyzed the features of manifestation and consequences of the formation of motor asymmetry, and assessed its impact on sports performance. Motor asymmetry has been found to be based on the genetic characteristics of the organism, but the lateral phenotype may change during a multi-year training process under the influence of physical exertion. Excessive asymmetry has been shown to cause various pathologies and injuries. The results of the study indicate that in aerobic cyclic sports motor asymmetry can be both a factor contributing to the growth of sports performance and a limiting one, depending on what function this or that part of the body performs. Despite the repetitive cycle of motor actions, typical for cyclic sports, only in 3 out of the 8 considered functions performed by limbs or a certain part of the body, motor asymmetry was a limiting factor. This allowed the authors to predict a possible conceptual scheme of motor asymmetry control in aerobic cyclic sports.
\end{abstract}

Keywords: motor asymmetry, cyclic sports, sports performance, sports training.

Research area: theory and methods of physical education, sports training, healthimproving and adaptive physical culture.

Citation: Khudik, S.S., Chikurov, A.I., Burmistrov, A.D., Voinich, A.L. (2021). Conceptual scheme of motor asymmetry control in aerobic cyclic sports. J. Sib. Fed. Univ. Humanit. Soc. Sci., 14(2), 214-225. DOI: $10.17516 / 1997-1370-0713$.

(C) Siberian Federal University. All rights reserved

* Corresponding author E-mail address: Zigi136@yandex.ru, Chikurov71@mail.ru, Burmistrovandre@yandex.ru, Aleksandr.

Voynich@mail.ru ORCID: 0000-0003-3450-8454 (Khudik); 0000-0003-0172-4898 (Voinich) 


\title{
Концептуальная схема управления моторной асимметрией в циклических видах спорта аэробной направленности
}

\author{
С.С. Худик, А.И. Чикуров, \\ А.Д. Бурмистров, А.Л. Войнич \\ Сибирский федеральный университет \\ Российская Федераџия, Красноярск
}

\begin{abstract}
Аннотация. В настоящей статье на основе известных литературных данных рассмотрена моторная асимметрия человека в тренировочном процессе циклических видов спорта аэробной направленности. Авторами проанализированы особенности проявления и следствия формирования моторной асимметрии, а также проведена оценка ее влияния на спортивный результат. Установлено, что моторная асимметрия формируется на основе генетических особенностей организма, однако в ходе многолетнего тренировочного процесса под воздействием физической нагрузки латеральный фенотип может изменяться. Выявлено, что чрезмерно выраженная асимметрия может повлечь за собой различные патологии и травмы. Результаты исследования свидетельствуют о том, что в циклических видах спорта аэробной направленности моторная асимметрия может являться фактором как способствующим росту спортивного результата, так и лимитирующим, в зависимости от того, какую функцию выполняет та или иная часть тела. Несмотря на повторяющийся цикл двигательных действий, характерный для циклических видов спорта, в трех из восьми рассмотренных функций, которые выполняют конечности или определенная часть тела, моторная асимметрия была лимитирующим фактором. Это обстоятельство позволило авторам спрогнозировать возможную концептуальную схему управления моторной асимметрией в циклических видах спорта аэробной направленности.
\end{abstract}

Ключевые слова: моторная асимметрия, циклические виды спорта, спортивный результат, спортивная подготовка.

Научная специальность: 13.00.04 - теория и методика физического воспитания, спортивной тренировки, оздоровительной и адаптивной физической культуры.

\section{Введение}

В циклических видах спорта повышение эффективности тренировочного процесса достигается в большинстве случаев через использование методик тренировок, направленных на улучшение показателей функциональной подготовленности спортсменов (Bykov et al., 2020; Golovachev et al., 2020), своевременную диагностику их физического утомления (Kornyakova et al., 2020), ускорение процессов восстановления и повышение уровня работоспособности (Shirkovets et al., 2019; Bondarenko, Martinovich, 2019). Эти методики являются обоснованными ввиду высоких требований к общей выносливости спортсменов в данных видах спорта, связанных с необходимостью преодолевать нагрузку продолжительное время - от 40 минут до нескольких часов. В некоторых методиках тренировки в циклических видах спорта используется акцентированное воздействие на определенные физические качества спортсменов (Golovachev et al., 2020; Mutaeva, Petrov, 2020), а также задействуются морфологические характеристики (Kovalchuk, Shastin, 2019; Shcheglov et al., 2020) и модельные показатели физических качеств, которы- 
ми должен обладать спортсмен, чтобы достигнуть запланированного спортивного результата (Golovachev et al., 2019). Формирование таких модельных характеристик позволяет определять в системе отбора наиболее результативных спортсменов в том или ином виде спорта (Kudriashova et al., 2019; Darenskaya et al., 2019; Sarsembayeva, 2019; Churikova, Sinelnikov, 2017; Yakushev, 2020).

Продолжительность и последовательность развития основных физических качеств в циклических видах спорта, безусловно, важнейшие элементы процесса спортивной подготовки. Однако если внимательно рассмотреть основное двигательное действие спортсменов, которое систематически повторяется в ходе тренировок, то можно заметить и другие значимые элементы. Так, например, можно увидеть, что выполнить двигательное действие технически одинаково с приложением равных усилий правой и левой сторон человеческого тела и его конечностей практически невозможно. Это обстоятельство объясняется наличием у каждого человека функциональной асимметрии (Bragina, Dobrokhodova, 1998).

Как известно, функциональная асимметрия изучается с момента открытия Броком центра речевой моторики в 1861 году (Broca, 1861) и на текущий момент находит свое отражение в различных системах организма. Функциональную асимметрию можно учитывать и при выборе методики обучения техническим действиям с соответствующим преобладанием заданий на ту или иную систему восприятия информации (внимание, восприятие, мышление, представление, воображение, память (Korobeinikov, et al., 2014; Anisimov, 2011)).

Выделяют основные виды функциональной асимметрии: моторную, психическую и сенсорную (Bragina, Dobrokhodova, 1998). В основе моторной асимметрии лежит наличие ведущей и вспомогательной конечностей при выполнении двигательного действия (Monastyrev, 2019). Поэтому у спортивных педагогов особый интерес вызывает исследование связи моторной асимметрии с двигательными способностями. В целом латеральный фенотип является фактором, определяющим успешность протекания процессов адаптации к спортивным нагрузкам, связанным с необходимостью быстрой реализации моторных программ (Fomina, 2006).

Сказанное выше означает, что моторная асимметрия может оказывать влияние как на выполнение двигательного действия, так и на всю работоспособность спортсмена. Недостаток системно изложенных сведений по вопросу использования моторной асимметрии в процессе спортивной подготовки в циклических видах спорта определил актуальность данной работы. Целью исследования являлась оценка ресурсных возможностей моторной асимметрии с точки зрения возможности улучшения спортивного результата в циклических видах спорта аэробной направленности. Задачи исследования включали: 1) изучение особенностей формирования и следствий проявления моторной асимметрии в циклических видах спорта аэробной направленности; 2) оценку влияния сформированной моторной асимметрии на спортивный результат в циклических видах спорта аэробной направленности; 3) прогнозирование концептуальной модели управления моторной асимметрией для улучшения спортивного результата в циклических видах спорта аэробной направленности.

\section{Методы исследования}

Моторная асимметрия человека и ее динамика в тренировочном процессе на примере циклических видов спорта аэробной направленности рассмотрены на основе известных в настоящее время литературных данных, как зарубежных, так и отечественных. Поиск источников проводился среди публикаций, размещенных в библиографических базах данных «Elibrary», «Scopus», «Web of Science», «Elsevier», «Российская Государственная Библиотека», посредством таких ключевых запросов, как «функциональная асимметрия», «моторная асимметрия», «циклические виды спорта», «подготовка в циклических видах спорта», 
«влияние функциональной асимметрии», «субмаксимальная мощность», «максимальная мощность», «зоны мощности», «аэробное энергообеспечение в циклических видах спорта», «asymmetry», «leg asymmetry». Полученные результаты были систематизированы в единую базу данных, в которой для каждого источника были зафиксированы основные результаты исследования. После этого был проведен отбор наиболее релевантных источников. Для решения задач исследования рассматривались основные виды дисциплин циклических видов спорта, на которых базируются смежные дисциплины и которым посвящено достаточное количество работ. Для прогнозирования концептуальной модели управления моторной асимметрией были выделены циклические виды спорта аэробной направленности.

Результаты и обсуждение

Особенности проявления

и следствия формирования

моторной асимметрии

в циклических видах спорта

Распределение функциональных показателей между полушариями заложено генетически. Однако при воздействии на них многочисленных факторов возможно их активное преобразование и изменение (Zubova, 2020). Некоторые исследователи в своих работах рассматривают взаимосвязь функциональной асимметрии с возрастом (Meyers et al., 2007; Sichko, Stalnaya, 2019; Pankova, Karganov, 2019; Ponomareva, Bushueva, 2019) и полом (Geodakian, 2005). На то, что неравенство рук и ног заложено на генетическом уровне, указывают многочисленные исследования Хемфри (Humphrey, 1861), Бирфлит (Van Biervliet, 1901), Стир (Stier, 1911), Масюк (Masyuk, 1939) и Поцелуева (Potseluev, 1960).

Под воздействием физической нагрузки у спортсменов запускаются адаптивные процессы, связанные с необходимостью быстрой реализации моторных программ (Fomina, 2006). Так, Игнатьева и Сергеев (Ignateva, Sergeev, 2019) установили, что среди спортсменов лыжников-гонщиков с ростом квалификации увеличивается число амбидекстров. Исследования Тришина (Trishin et al., 2015) свидетельствуют о закреплении латеральных предпочтений у спортсменов в соответствии с характером нагрузок в избранном виде спорта.

Таким образом, латеральный фенотип спортсмена, заложенный на генетическом уровне, меняется в течение всего тренировочного процесса и с ростом квалификации моторная асимметрия либо сглаживается под воздействием одинаковых двигательных действий, выполняемых конечностями, либо, напротив, усиливается. Однако в условиях возрастающей нагрузки в многолетнем тренировочном процессе выраженная моторная асимметрия становится фактором, провоцирующим возникновение спортивных травм. Об этом свидетельствует множество исследований (Gore et al., 2014; Abramova et al., 2013; Sedochenko, 2015; Shevtsov, 2012; Knapik et al., 1991; Poluektov, 2013; Plotnikov, Mar'ianovskii, 2007). Эффективной профилактикой травматизма является управление тренировочным процессом с учетом моторной асимметрии (Ignatieva, Chetaykina, 2019) и усиление врачебного контроля для прогнозирования нарушений опорно-двигательного аппарата, возможности коррекции и профилактики заболеваний (Motova, Oleinik, 2020).

\section{Влияние моторной асимметрии на результат в циклических видах спорта аэробной направленности}

Результаты многочисленных исследований показывают актуальность изучения взаимосвязи моторной асимметрии и спортивного результата. Однако однозначный тезис, характеризующий положительное или отрицательное влияние асимметрии, для всех дисциплин циклических видов спорта сформулировать нельзя, так как двигательные действия в каждой дисциплине выполняются в различных зонах относительной мощности, различающихся по продолжительности работы. Среди циклических видов спорта В.С. Фарфел выделяет четыре зоны относительной мощ- 
ности: максимальную, субмаксимальную, большую и умеренную (Farfel, 1969). Зона мощности напрямую отражается на биомеханических характеристиках двигательных действий, а также на источниках энергообеспечения. Для видов спорта, работа которых относится к большой и умеренной мощности, характерен аэробный режим энергообеспечения (Baranova, 2012). В зависимости от мощностного режима поразному может проявляться и моторная асимметрия. Об этом свидетельствует исследование Л.П. Черапкиной, в котором сообщается, что по мере утомления моторная асимметрия увеличивается, так как на неведущей конечности быстрее происходит падение силы при длительной активности (Cherapkina, 2005).

В современной литературе есть сведения как о положительном, так и об отрииательном влиянии моторной асимметрии на спортивный результат. Например, асимметричность рук пловиов оказывает прямое влияние на длину, силу и качество гребков. Качество гребка ведущей рукой на вдохе более эффективно, чем при дыхании в субдоминантную сторону. Это говорит о необходимости выявления моторной асимметрии рук на первых занятиях и ее учета при разучивании способа плавания кролем (Gramatikopolo, 2011). При изучении биомеханики движения конькобежца установлено, что наличие асимметричного смещения центра масс тела конькобежцев при выполнении отталкивания правой и левой конечностями на прямых участках дистанции влечет за собой увеличение продолжительности выполнения одной из конечностей свободного проката и одноопорного отталкивания (Burmistrov et al., 2018).

В ходе обзора литературы обнаружены результаты исследований, в которых для одного и того же вида спорта моторная асимметрия является как фактором, способствующим росту спортивного результата, так и лимитирующим фактором. Например, исследования Stöggl, который установил, что при выполнении одновременного двухшажного хода спортсменами в льжном спорте (горный стиль) толчок более эффективен, если он выполнен на ведущую сторону. Кроме того, эта эффективность возрастает с увеличением интенсивности выполнения двигательного действия спортсмена (Stöggl et al., 2013). Однако направленность тренировочного процесса, ориентированная на сглаживание асимметрии действий в лыжных гонках классическим стилем, оказывает положительное влияние на спортивный результат. Так, большинство лыжниковгонщиков в группе мастеров спорта являются амбидекстрами (Ignateva, Sergeev, 2019).

Карпеса выявил среди профессиональных велогонщиков то, что асимметрия в работе их ног присутствует и имеет устойчивую зависимость от интенсивности педалирования - чем выше интенсивность, тем ниже показатель асимметрии (Carpes et al., 2007). В то же время González-Sánchez и др. установили, что показатель асимметрии между правой и левой ногами увеличивается при педалировании в положении стоя, с увеличением интенсивности и мощности нажима ногами (González-Sánchez et al., 2019).

В гребле на байдарках увеличение асимметрии изгиба поясничного отдела позвоночника во фронтальной плоскости способствует увеличению гоночной скорости (Rynkiewicz et al., 2013), в то время как уменьшение коэффициента асимметрии для верхних и нижних конечностей в гребле на байдарках, проявляющееся в неравнозначности прилагаемых усилий на лопасть весла и на подножку у гребцовбайдарочников, приводит к увеличению скорости преодоления дистанции (Bryukhanov, Kornilov, 2014).

Сводные результаты оценки влияния моторной асимметрии на спортивный результат в циклических видах спорта аэробной направленности приведены в табл. 1.

Таким образом, согласно данным табл. 1, в циклических видах спорта аэробной направленности моторная асимметрия может быть фактором, как способствующим росту спортивного результата, так и лимитирующим, в зависимости от того, 
Таблица 1. Влияние моторной асимметрии (МА) на спортивный результат в циклических видах спорта аэробной направленности

Table 1. Influence of motor asymmetry (MA) on sports scores in cyclic aerobic sports

\begin{tabular}{|c|c|c|c|c|}
\hline $\begin{array}{l}\text { № } \\
\text { П/П }\end{array}$ & Вид спорта & $\begin{array}{l}\text { Часть тела, } \\
\text { проявляю- } \\
\text { щая МА }\end{array}$ & Влияние МА на спортивный результат & $\begin{array}{c}\text { Оценка } \\
\text { влияния МА } \\
\text { на спортив- } \\
\text { ный результат } \\
\text { «+», «-» }\end{array}$ \\
\hline 1 & Плавание & $\begin{array}{l}\text { верхние } \\
\text { конечности }\end{array}$ & $\begin{array}{l}\text { увеличение длины, силы и качества гребков } \\
\text { (Gramatikopolo, 2011) }\end{array}$ & + \\
\hline 2 & $\begin{array}{l}\text { Конькобеж- } \\
\text { ный спорт }\end{array}$ & $\begin{array}{l}\text { нижние } \\
\text { конечности }\end{array}$ & $\begin{array}{l}\text { увеличение продолжительности свободно- } \\
\text { го проката и одноопорного отталкивания } \\
\text { (Burmistrov, Chikurov, Khudik, Radaeva, 2018) }\end{array}$ & + \\
\hline 3 & $\begin{array}{l}\text { Лыжный спорт. } \\
\text { Классический ход }\end{array}$ & $\begin{array}{l}\text { верхние } \\
\text { конечности }\end{array}$ & $\begin{array}{l}\text { снижение гоночной скорости (Ignateva, } \\
\text { Sergeev, 2019) }\end{array}$ & - \\
\hline 4 & $\begin{array}{l}\text { Лыжный спорт. } \\
\text { Коньковый ход }\end{array}$ & $\begin{array}{l}\text { верхние } \\
\text { конечности }\end{array}$ & $\begin{array}{l}\text { возрастание эффективности отталкивания } \\
\text { (Stöggl, Hébert-Losier, Holmberg, 2013) }\end{array}$ & + \\
\hline 5 & Велоспорт & $\begin{array}{l}\text { нижние } \\
\text { конечности }\end{array}$ & $\begin{array}{l}\text { увеличение интенсивности и мощности пе- } \\
\text { далирования в положении стоя (González- } \\
\text { Sánchez, Barranco-Gil, Fernández-Luna, } \\
\text { Felipe, García-Merino, Barbado-Villalba, 2019) }\end{array}$ & + \\
\hline & & & $\begin{array}{l}\text { увеличение интенсивности педалирования } \\
\text { (Carpes, Rossato, Faria, Mota, 2007) }\end{array}$ & - \\
\hline 6 & Гребля на бай- & $\begin{array}{l}\text { пояснич- } \\
\text { ный отдел }\end{array}$ & $\begin{array}{l}\text { увеличение гоночной скорости (Rynkiewicz, } \\
\text { Rynkiewicz, Starosta, 2013) }\end{array}$ & + \\
\hline 0 & дарках & $\begin{array}{l}\text { верхние } \\
\text { конечности }\end{array}$ & $\begin{array}{l}\text { увеличение скорости преодоления дистан- } \\
\text { ции (Bryukhanov, Kornilov, 2014) }\end{array}$ & - \\
\hline \multicolumn{4}{|c|}{ ИТОГО } & $5 \ll+» ; 3 \ll-»$ \\
\hline
\end{tabular}

какую функцию выполняет та или иная часть тела. Несмотря на повторяющийся цикл двигательных действий, характерный для циклических видов спорта, в трех из восьми рассмотренных функций, которые выполняют конечности или определенная часть тела, моторная асимметрия является лимитирующим фактором. Это обстоятельство позволяет спрогнозировать концептуальную схему управления моторной асимметрией в циклических видах спорта аэробной направленности.

\section{Концептуальная схема управления моторной асимметрией}

На основе вышеприведенного обзора формализуем концептуальную схему управления моторной асимметрией, отражающую ее взаимосвязь с тренировочной нагрузкой и спортивным результатом в циклических видах спорта аэробной направленности (рис. 1).

Как видно на рис. 1, на начальном этапе спортивной подготовки в циклических видах спорта (стадия 1) происходит опосредованное управление моторной асимметрией - через спортивную нагрузку. На данном этапе функциональная асимметрия, имеющая исключительно генетическую основу, является значимым фактором спортивной подготовки, однако практически не учитывается в тренировочном процессе. На последующем этапе (стадия 2) после того, как в процессе многолетней тренировки на базе генетических особенностей спортсмена сформировалась индивидуальная и обусловленная занятиями тем или иным видом спорта моторная асимметрия, она может быть использована в построении тренировочного процесса. В конечном счете, мы 

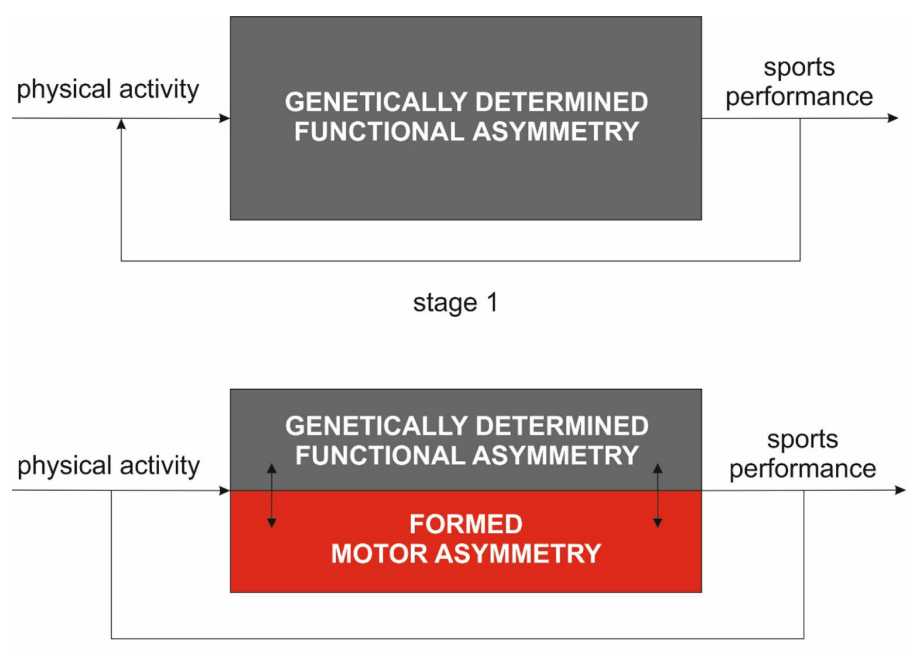

stage 2

Рис. 1. Концептуальная схема управления моторной асимметрией в циклических видах спорта аэробной направленности

Fig. 1. Conceptual scheme of motor asymmetry control in cyclic aerobic sports

предполагаем, что это может позволить улучшить спортивный результат.

\section{Заключение}

Латеральный фенотип спортсмена, заложенный на генетическом уровне, меняется в течение всего тренировочного процесса как в сторону усиления, так и в сторону сглаживания. Необходимо учесть, что выраженная моторная асимметрия может оказывать негативное влияние на опорнодвигательный аппарат и быть фактором, провоцирующим возникновение спортивных травм. В этой связи целесообразно проводить реабилитационные мероприятия, направленные на коррекцию выявляемых нарушений.

В циклических видах спорта аэробной направленности моторная асимметрия может выступать как фактором, способствующим росту спортивного ре- зультата, так и лимитирующим, в зависимости от того, какую функцию выполняет та или иная часть тела. Несмотря на повторяющийся цикл двигательных действий, характерный для циклических видов спорта, лишь в трех из восьми рассмотренных функций, которые выполняют конечности или определенная часть тела, моторная асимметрия является лимитирующим фактором.

В результате исследования представлена концептуальная схема управления моторной асимметрией в циклических видах спорта аэробной направленности, отражающая взаимосвязь моторной асимметрии с тренировочной нагрузкой и спортивным результатом. Исследование дает возможность заключить, что моторная асимметрия должна учитываться при управлении тренировочным процессом с целью улучшения спортивного результата.

\section{Список литературы / References}

Abramova, T.F., Nikitina, T.M., Kochetkova, N.I., Krasnikov, V.A. (2013). Osobennosti prostranstvennogo polozheniia tulovishcha, taza i stop u vysokokvalifitsirovannykh sportsmenov-muzhchin razlichnykh vidov sporta [Features of the spatial position of the trunk, pelvis and feet in highly skilled male athletes of various sports]. In Vestnik sportivnoy nauki [Sports science bulletin], 5, 58-65. 
Ammann, R., Wyss, T. (2015). Running asymmetries during a 5-km time trial and their changes over time. In Proceedings ofthe 3rd International Congress on Sport Sciences Research and Technology Support, 1, 161-164.

Anisimov, M.P. (2011). Ob uchenie tekhnicheskim deistviyam v smeshannykh edinoborstvakh $\mathrm{s}$ uchetom mezhpolusharnoi asimmetrii [Trainingin technical actions in mixed martial arts taking into account interhemispheric asymmetry]. In Uchenye zapiski universiteta imeni P.F. Lesgafta [Scientific notes of the University of P.F. Lesgafta], 1, 426-428.

Baranova, T.I. (2012). Fiziologicheskie osnovy fizicheskogo vospitaniia [Physiological basis of physical education]. Saint Petersburg, Sankt-peterburgskii gosudarstvennyi universitet, 35 p.

Bobina, O.N. (2007). Eksperimental'noe obosnovanie metodicheskikh priemov v obuchenii dvigatel'nym deistviyam s uchetom motornykh asimmetrii [Experimental substantiation of methods in teaching motor actions with account for motor asymmetries]. In Vestnik TGPU [TSPU Bulletin], 5(68), $28-30$.

Bondarenko, A., Martinovich, S. (2019). Issledovanie mekhanizma «srochnoi» adaptatsii organizma sportsmenov tsiklicheskikh vidov sporta [Study of the mechanism of the «urgent» adaptation of the sportsmen's organism of cyclic sports], In Norwegian journal of development of the international science, 4(29), 21-23.

Bragina, N.N., Dobrokhodova, T.A. (1998). Funktsional'nye asimmetrii cheloveka [Functional asymmetric of human]. Moscow, Meditsinskie otnosheniia, $238 \mathrm{p}$.

Broca, P.P. (1861). Perte de la parole, ramollissement chronique et desstruction partielle du lob antérieur gauche de cerveau [Loss of speech, chronic softening and partial destruction of left anterior brain lob]. In Bulletins de la Société d'Anthropologie, 2, 235-238.

Bryukhanov, D.A., Kornilov, Yu.P. (2014). Sovershenstvovanie dvigatel'nykh deistviy grebtsov s uchetom asimmetrii spetsial'nykh silovykh kachestv [Perfection of the motor actions of oarsmen taking into account the asymmetry of special strength qualities]. In Izvestiia Sochinskogo gosudarstvennogo universiteta [Sochi Journal of Economy], 1 (29), 222-225.

Burmistrov, A. D., Chikurov, A. I., Khudik, S. S., Radaeva, S. V. (2018). Funkstional'naia asimmetriia kon'kobezhtsev vysokoi kvalifikatsii [Functional asymmetry of elite speed skaters]. In Vestnik Tomskogo gosudarstvennogo universiteta [Tomsk State University Journal], 434, 143-148. DOI: $10.17223 / 15617793 / 434 / 19$.

Bykov, E.V., Balberova, O.V., Chipyshev, A.V., Sidorkina, E.G. (2020). Osobennosti funktsyonal'noi podgotovlennosti sportsmenov tsiklicheskikh vidov sporta c raznoi spetsifikoi trenirovochnogo protsessa [Features of the Functional Preparedness of Athletes in Cyclic Sports with Different Specifics of the Training Process]. In Vestnik MGPU. «Estestvennye nauki» [MGPU Bulletin natural sciences], 2(38), 78-89. DOI: $10.25688 / 2076-9091.2020 .38 .2 .7$.

Carpes, F.P., Rossato, M., Faria, I.E., Mota, C.B. (2007). Bilateral pedaling asymmetry during a simulated 40-km cycling time-trial. In Journal of Sports Medicine and Physical Fitness, 47, 51-57.

Cherapkina, L.P. (2005). Medikobiologicheskie osnovy otbora i prognozirovaniia vysshikh sportivnykh dostizhenii [Medical and biological basis of selection and prediction of higher sports achievements]. Omsk, SibGUFK, $72 \mathrm{p}$.

Churikova, L.N., Sinelnikov, M.S. (2017). Otbor I orientatsiia v tsiklicheskikh vidakh sporta (na primere lyzhnykh gonok) [Selection and orientation in cyclic sports (on the example of ski racing)]. In $\mathrm{Me}$ dikobiologicheskie I pedagogicheskie osnovy adaptatsii sportivnoi deiatel'nosti I zdorovogo obraza zhizni [Medical-biological and pedagogical basis of adaptation, sports activities and healthy lifestyle]. Voronezh, 369-373.

Darenskaya, M.A., Alexandrov, S.G., Suslikova, M.I., Grebenkina, L.A., Gubina, M.I., Kolesnikova, L.I. (2019). Raspredelenie lateralnykh sensomotornykh priznakov u studentov meditsinskogo universiteta [The distribution of lateral sensorimotor characters among the Students of medical university]. In Sovremennye problemy nauki i obrazovaniia [Modern problems of science and education], 5. DOI:10.17513/ spno.29226. 
Farfel, V.S. (1969). Fiziologicheskie osnovy klassifikatsii fizicheskikh uprazhneniy [Physiological basis of classification of physical exercises]. In Rukovodstvo po fiziologii fiziologiya myshechnoy deyatelnosti truda I sporta [Guidelines for Physiology. Physiology of muscle activity, work and sport], 425-439.

Fomina, E.V. (2006). Funktsional'naia asimmetriia mozga i adaptatsiia cheloveka k ekstremal'nym sportivnym nagruzkam [Functional asymmetry of the brain and adaptation of a human to extreme sports loads]. In Extended abstract of biol. Sci. Tyumen, 329.

Geodakian, V.A. (2005). Evolutionary theories of asymmetrization of organisms, brain and body. In Uspekhi Fiziologicheskikh Nauk [Advances in Physiological sciences], 36(1), 24-53.

Golovachev, A.I., Kolykhmatov, V.I., Shirokova, S.V. (2019). Model'nye pokazateli fizicheskoi podgotovlennosti lyzhnits-gonshchits vysokoi kvalifikatsii pri podgotovke k XXIV Zimnim olimpiiskim igram 2022 goda v Pekine (Kitai) [Model indicators of physical area ski racers of high qualification in preparation for the xxiv winter olympic games 2022 in beijing (china)]. In Chelovek. Sport. Meditsina [The Human. The Sport. The medicine], 2, 81-87. DOI: 10.14529/hsm19s211.

Golovachev, A.I., Kolykhmatov, V.I., Shirokova, S.V. (2020). Vliianie maksimal'noi anaerobnoi (glikoliticheskoi) proizvoditel'nosti na effektivnost' vistupleniia visokokvalifitsirovannykh lyzhnits-gonshchits v sorevnovaniiakh na razlichnykh distantsiiakh [Influence of maximum anaerobic (glycolytic) performance on the sports result of elite cross-country female skiers in competitions at various distances]. In Uchenye zapiski unversiteta imeni P.F. Lesgafta [Scientific notes of the University of P.F. Lesgafta], 5 (183), 96-102. DOI: $10.34835 /$ issn.2308-1961.2020.5.

Golovachev, A.I., Kolykhmatov, V.I., Shirokova, S.V. (2020). Vliianie vzryvnoi sily nog na effektivnost' vystupleniia vysokokvalifitsirovannykh lyzhnits-gonshchits $v$ sorevnovaniiakh na razlichnykh distantsiiakh [Influence of the explosive force of the legs on the efficiency of performance highly qualified racing skiers in competition at different distances], In Uchenye zapiski universiteta imeni P.F. Lesgafta [Scientific notes of the University of P.F. Lesgafta], 5(183), 90-96.

González-Sánchez, J., Barranco-Gil, D., Fernández-Luna, Á., Felipe, J.L., García-Merino, S., Barbado-Villalba, C. (2019). Impact of rider position and pedaling cadence on power output and bilateral asymmetry in indoor cycling. In Journal of Sports Medicine and Physical Fitness, 59(12), 2009-2014.

Gore, S., Richter, C., Marshall, B., Franklyn-Miller, A., Kieran, M., Blanchfield, M., Moore, B., Falvey, E. (2014). A comparison of asymmetry in athletic groin pain patients and elite rugby union players using analysis of characterizing phases. In International Conference of Biomechanics in Sports, Johson City, TN, USA. $237-240$.

Gramatikopolo, S.N. (2011). Vliianie funktsional'noi asimmetrii na kachestvo grebkov u iunykh plovtsov 8-10 let [Influence of functional asymmetry on the quality of strokes in young swimmers aged 8-10]. In Vestnik sportivnoi nauki [Sports science bulletin], 2, 28-30.

Gramatikopolo, S.N. (2011). Vliianie funktsional'noy asimmetrii na kachestvo grebkov u iunykh plovtsov 8-10 let [Influence of functional asymmetry on the quality of strokes in young swimmers aged 8-10]. In Vestnik sportivnoy nauki [Sports science bulletin], 2, 28-30.

Humphrey, G.M. (1861). The human foot and the human hand. Cambridge, England, Macmillan \& Co., $215 \mathrm{p}$.

Ignateva, L.E., Sergeev, M.G. (2019). Prognozirovanie rezul'tativnosti lyzhnikov- gonshchikov na osnove ucheta individual'nogo profilia asimmetrii [Predicting the performance of skiers-racers based on taking into account the individual asymmetry profile]. In Globalnyi nauchnyi potentsial [Global scientific potential], 12(105), 94-97.

Ignateva, L.E., Sergeev, M.G. (2019). Prognozirovanie rezul'tativnosti lyzhnikov- gonshchikov na osnove ucheta individual'nogo profilia asimmetrii [Predicting the performance of skiers-racers based on taking into account the individual asymmetry profile]. In Globalnyi nauchnyi potentsial [Global scientific potential], 12(105), 94-97.

Ignatieva, L.E., Chetaykina, O.V. (2019). Issledovanie funktsional'noi motornoi asimmetrii lyzhnikov gonshchikov $\mathrm{v}$ aspekte profilaktiki travmatizma [Research of functional motor asymmetry of ski-racers in the aspect of prevention of injuries]. In Kultura fizicheskaia i zdorove [Physical culture and health], 4(72), 62-64. 
Khudik, S., Chikurov, A., Petrova M., Burmistrov, A. (2018). Functional asymmetry and the sports result. In Journal of Physical Education and Sport, 1(57), 408 - 415. DOI:10.7752/jpes.2018.s157.

Knapik, J.J., Bauman, C.L., Jones, B.H., Harris, J.M., Vaughan, L. (1991). Preseason strength and flexibility imbalances associated with athletic injuries in female collegiate athletes. In American Journal of Sports Medicine, 19 (1), 76-81. DOI: 10.1177/036354659101900113.

Kornyakova, V.V., Badtieva, V.A., Conway, V.D. (2020). Funktsional'naia gotovnost' sporsmenov tsiklicheskikh vidov sporta [The Functional Readiness of Athletes from Cyclic Sports]. In Chelovek. Sport. Meditsina [Human. Sport. Medicine], 20 (1), 128-134. DOI: 10.14529/hsm200116.

Korobeinikov, G.V., Korobeinikova, L.G., Mishchenko, V.S., Rychok, T.M. (2014). Funktsional'naia mezhpolusharnaia asimmetriia mozga i kognitivnye funktsii u elitnykh bortsov [Functionalinterhemispheric asymmetry of the brain and cognitive functions in elite wrestlers]. In Voprosy funktsional'noy podgotovki $v$ sporte vysshikh dostizheniy [Functional training in elite sports], 2, 53-63.

Kovalchuk, G.I., Shastin, A.A. (2019). Morfotipologicheskie kriterii otbora i podgotovki v tsiklicheskikh vidakh sporta [Morphotypological criteria for selection and preparation in cyclic sports]. In Modern science, 4-3, 87-89.

Kudriashova, Y.A., Rovnyi, D.A., Berdichevskaia, E.M., Rodionov, S.S. (2019). Osobennosti funktsional'nykh asimmetrii rezul'tativnykh sportsmenov-vaterpolistov [Features of functional asymmetries effective athletes-water-players], In Resursy konkurentosposobnosti sportsmenov teoriia I praktika realizatsii [Resources of athletes 'competitiveness: theory and practice of implementation], 1, 188-189.

Lavrent'eva, D.A. (2016). Osobennosti rezul'tatov sorevnovatel'noi deiatel'nosti plovtsov 10-12 let s raznymi tipami profilei motornoi asimmetrii [Features of the results of competitive activities of swimmers aged 10-12 with different types of motor asymmetry profiles]. In Vestnik Adygeiskogo gosudarstvennogo universiteta [Bulletin of the Adygeya State University], 2(178), 125-132.

Lavrent'eva, D.A. (2015). Nachal'noe obuchenie plavaniiu detei mladshego shkol'nogo vozrasta s uchetom motornykh asimmetrii [Initial training in swimming for children of primary school age with account for motor asymmetries]. Extended abstract of Cand. Sci. Dissertation of ped. Sci. Malakhovka, 24.

Masyuk, A.I. (1939). Korregirovanie funktsional'noy asimmetrii fizicheskimi uprazhneniyami kak metod povysheniya sportivno-tekhnicheskikh rezul'tatov [Correlation of functional asymmetry in physical exercises as a method of improving sports and technical results]. In Trudy Ukrainskogo nauchno-issledovatel'skogo instituta fizicheskoi kul'tury [Proc. research institute of physical culture of Ukraine], 145-156.

Meyers, R.W., Oliver, J.L., Hughes, M.G., Lloyd, R.S., Cronin, J.B. (2007). Asymmetry during maximal sprint performance in 11- to 16-year-old boys. In Pediatric Exercise Science, 29, 94-102. DOI: 10.1123/ pes.2016-0018.

Monastyrev, S.N. (2019). Kriterii oopredeleniia vedushchei (tolchkovoi) nogi s uchetom pokazatelei motornoi asimmetrii nizhnikh konechnostei u sportsmenov razlichnoi spetsializatsii I urovnia podgotovlennosti [Criteria for determining the dominant (take-off) foot in the contaxt of legs' motor asymmetry of athletes in various sports and with different functional level]. In Uchenye zapiski universiteta imeni P.F. Lesgafta [Scientific notes of the University of P.F. Lesgafta], 8(174), 150-154.

Motova, E.A., Oleinik, E.A. (2020). Vliianie sportivnoi deiatel'nosti na formirovanie morfofunktsional'noi asimmetrii u figuristov odinochnikov 12-15 let [Impact of sporting activities on formation of morphofunctional asymmetries in lone figurists 12-15 years old]. In Konkurs nauchnykh innovatsii perspektivy razvitiia nauki $v$ sovremennom mire [Competition of scientific innovations: prospects for the development of science in the modern world]. Ufa, 6-11.

Mutaeva, I.S., Petrov, R.E. (2020). Posledovatelnost' i prodolzhitelnost' razvitiia fizicheskikh kachestv $\mathrm{v}$ tsiklicheskikh vidakh sporta s proiavleniem vynoslivosti [Sequence and duration of development of physical qualities in cyclic sports with endurance]. In Pedagogiko-psikhologicheskie i medico-biologicheskie problemy fizicheskoi kultury i sporta [Pedagogical-psychological and medical-biological problems of physical culture and sports], 1(15), 41-47.

Pankova, N.B., Karganov, M.Y. (2019). Vzaimosviaz' razlichnykh pokazatelei motornoi asimmetrii ruk u pervoklassnikov obsledovannykh $\mathrm{v}$ dinamike uchebnogo goda [Interconnection of different indicators 
motor asymmetry of hands in accommodations, examined in the dynamics of the school year]. In Psikhologiia. Psikhofiziologiia [Psychology. Psychophysiology], 2(12), 72-79.

Plotnikov, S.G. (2018). Mediko-fiziologicheskie osobennosti monitoring funktsional'nogo sostoianiia lyzhnikov s razlichnym profilem motornoi asimmetrii [Medical and physiological features of monitoring the functional state of skiers with different profiles of motor asymmetry]. In Extended abstract of Cand. Sci. Dissertation of med. Sci. Moscow, 24.

Plotnikov, S.G., Mar'ianovskii, A.A. (2007). Funktsional'noe sostoianie elitnykh sportsmenov-lyzhnikov s uchetom dvigatel'noi asimmetrii [Functional state of elite skiers with account for motor asymmetry]. In Teoriya i praktika fizicheskoy kul'tury [Theory and Practice of Physical Culture], 1, 42-45.

Poluektov, E.S. (2013). Vliianie fizicheskikh nagruzok na sostoianie oporno-dvigatel'nogo apparata begunov na srednie distantsii [Influence of physical loads on the condition of the locomotor system of medium distance runners]. In Uchenye zapiski universiteta imeni P.F. Lesgafta [Scientific notes of the University of P.F. Lesgafta], 12 (106), 120-123. DOI: 10.5930/issn.1994-4683.2013.12.106.p120-123.

Ponomareva, T., Bushueva, T. (2019). Vozrastnye osobennosti funktsional'noi mezhpolusharnoi assimetrii nauchnyi aspect [Age peculiarities of functional interhemispheric asymmetry: the scientific aspect]. In Nauka 2020 [Science-2020], 8(33), 94-101.

Potseluev, A.A. (1960). Asimmetriya dvizheniy [Asymmetry of movements]. In Teoriya i praktika fizicheskoy kul'tury [Theory and Practice of Physical Culture], 7, 496-498.

Rynkiewicz, M., Rynkiewicz, T., Starosta, W. (2013). Asymmetry of Spinal Segments Mobility in Canoeists and its Relationship with Racing Speed. In Human Kinetics, 36, 37-43.

Sedochenko, S.V. (2015). Pedagogicheskaia korrektsiia asimmetrichnoi nagruzki u iunykh sportsmenov na osnove primeneniia sredstv srochnoi informatsii (na primere fekhtovaniia i tennisa) [Pedagogical correction of asymmetric load in young athletes based on the use of urgent information (in fencing and tennis)], Extended abstract of ped. Sci. Moscow, 184.

Shcheglov, V.N., Mashchenko, R.M., Iagodnitsyna, A.A. (2020). Metodika silovoi podgotovki zhenshchin, treniruiushchikhsia $\mathrm{v}$ tsiklicheskikh vidakh sporta, trebuiushchikh proiavleniia vynoslivosti [Method of power training of women, training in cyclic sports, requiring endurance]. In Medikobiologicheskie I pedagogicheskie osnovy adaptatsii sportivnoi deiatel'nosti I zdorovogo obraza zhizni [Medical-biological and pedagogical basis of adaptation, sports activities and healthy lifestyle]. Voronezh, 378-375.

Shevtsov, A.V. (2012). Funktsional'noe sostoianie vistseral'nykh sistem organizma sportsmenov pri nemedikamentoznom sposobe korrektsii myshechno-tonicheskoi asimmetrii paravertebral'noy zony [Functional state of visceral systems of the body of athletes with non-pharmacological method of correction of muscular-tonic asymmetry of the paravertebral zone]. Extended abstract of biol. Sci. Chelyabinsk, 323.

Shirkovets, E.A., Morozov, V.N., Rybina, I.L. (2019). Razlichie struktur funktsional'nykh pokazatelei sportsmenov $\mathrm{v}$ tsiklicheskikh vidakh sporta [Difference of structures of functional indicators in cyclic sports athletes]. In Vestnik sportivnoi nauki [Sports science bulletin], 3, 28-31.

Sichko, N.O., Stalnaya, M.I. (2019). Izmeneniia motornykh asimmetrii i ikh sviaz s mezhpolusharnoi funktsional'noi asimmetriei [Variations of motor asymmetries and their relationship with interhemescope functional asymmetry]. In Fundamental'nye I prikladnye nauki segodnia [Fundamental and applied sciences today]. North Charleston, 39-42.

Sousa, F., Rodrigues, N., Manchado-Gobatto, F., Gobatto, C. (2020). Effect of Running Intensity on Leg Force Asymmetry and its Relationship to Internal Load Biomarkers. In 2020 IEEE International Workshop on Metrology for Industry 4.0 and IoT, MetroInd 4.0 and IoT 2020 - Proceedings. Rome, 495-500.

Stier, E. (1911). Studies on left-handedness and the functional differences of the cerebral hemispheres [Untersuchungen über Linkshändigkeit und die funtionellen Differenzen der Hirnhälften], Jena, G. Fischer, $349 \mathrm{p}$.

Stöggl, T., Hébert-Losier, K., Holmberg, H.C. (2013). Do Anthropometrics, Biomechanics, and Laterality Explain V1 Side Preference in Skiers? In Med. Sci. Sports Exerc., 45 (8), 1567-1576. 
Sarsembayeva, E.Y., (2019). Funktsional'naia asimmetriia mozga I ee rol' v uspeshnosti sportivnoi deiatel'nosti [Functional brain asymmetry and its role in the success of sports activities]. In Materialy vserossiiskoi nauchno-prakticheskoi konferentsii «nauka i sotsium» [Materials of the all-Russian scientific and practical conference "science and society"]. Novosibirsk, 92-97.

Trishin, A.S., Trishin, E.S., Berdichevskaya, E.M., Katrich, L.V. (2015). Osobennosti portural'nogo rjynhjkia u vysokokvalifitsirovannykh sportsmenov v situatsionnykh vidakh sporta pri vozdeistvii kateralizovannykh faktorov [Features of postural factors during exposure to lateralized controls in athletes skilled at situational sports]. In Asimmetriia [Journal of Asymmetry], 1 (9), 4-11.

Vagenas, G., Hoshizaki, B. (2016). A Multivariable Analysis of Lower Extremity Kinematic Asymmetry in Running. In International Journal of Sport Biomechanics, 8, 11-29.

Van Biervliet, J.J. (1901). Études de psychologie [Psychology research]. Gand, 201 p.

Yakushev, D.S. (2020). Metodika sportivnogo otbora I rannei orientatsii detei 8-10 let v tsiklicheskie vidy sporta [Methods of sports selection and early orientation of children aged 8-10 years in cyclic sports]. In Izvestiia Tul'skogo gosudarstvennogo universiteta. Fizicheskaia kul'tura.Sport [Izvestiya Tula State University], 7, 130-135.

Zubova, K.Y. (2020). Funktsional'naia mezhpolusharnaia asimmitriia I ee vliianie na individual'nye osobennosti [Functional inter-hemispheric asymmetry and its influence on individual features]. In Nauchnaia initsiativa $v$ psikhologii [Scientific authority of psychology], 107-114. 